\title{
The relationship between the symptoms of female gonococcal infections and serum progesterone level and the genotypes of Neisseria gonorrhoeae multi-antigen sequence type (NG-MAST) in Wuhan, China
}

\author{
Z. Wu • L. Xu • Y. Tu • R. Chen • Y. Yu • J. Li • M. Tan • \\ H. Chen
}

Received: 9 March 2010 / Accepted: 4 August 2010 /Published online: 4 September 2010

(C) The Author(s) 2010. This article is published with open access at Springerlink.com

\begin{abstract}
The objective of this investigation was to study the relationship between the symptoms of female gonococcal infections and serum progesterone level and the genotypes of Neisseria gonorrhoeae multi-antigen sequence type (NG-MAST) in Wuhan, China. Eighty-one strains of $N$. gonorrhoeae were harvested from the vaginal discharge of 975 adult females in Wuhan and were genotyped by using NG-MAST. Serum progesterone (P) and estradiol $\left(\mathrm{E}_{2}\right)$ levels were measured by radio immunoassay (RIA) in 39 gonorrhea-infected patients with slight symptoms (asymptomatic group) and 42 patients with conspicuous symptoms (symptomatic group). The average levels of serum progesterone in the asymptomatic group were significantly higher than in the symptomatic group $(p<0.05)$, while no significant difference was found in serum estradiol between the two groups. Of 81 wild-type isolates, $50 \mathrm{NG-}$ MAST sequence types were associated with female infections in Wuhan, and N. gonorrhoeae ST2951, ST735, and ST436 were principally found in asymptomatic patients. ST809 and ST369, however, were mainly detected in asymptomatic female subjects. Gonococcal genetic island (GGI)-positive and GGI-negative strains were found in both
\end{abstract}

Zhihong $\mathrm{Wu}, \mathrm{Li} \mathrm{Xu}$, and Yating Tu contributed equally to this work.

L. Xu $\cdot$ Y. Tu $\cdot$ R. Chen $\cdot$ Y. Yu $\cdot$ J. Li $\cdot$ M. Tan $\cdot$ H. Chen $(\bowtie)$

Department of Dermatology, Affiliated Union Hospital,

Tongji Medical College,

Huazhong University of Science and Technology,

Wuhan 430022, China

e-mail:wslhss@126.com

Z. Wu

Department of Dermatology, Tianyou Hospital

Affiliated to Wuhan University of Science and Technology,

Wuhan 430064, China the asymptomatic group and the symptomatic group. In females with gonococcal infection, high serum progesterone level is associated with the absence of symptoms, but no association was revealed between genotypes and the presence of symptoms. The GGI bears no relation to the absence of symptoms in the patients.

\section{Introduction}

Gonorrhea is the most common sexually transmitted bacterial infection in China. Recent studies indicate that molecular mechanisms of the infection vary with anatomical sites [1]. In male patients and a few female patients, gonorrhea is characterized by purulent discharge (infiltration of polymorphonuclear neutrophils), but in most female patients, the symptoms of genital tract infection, such as pus discharge, abdominal pain, and dyspareunia, tend to be slight or even absent.

With a number of genital infections, sex hormones are associated with the susceptibility to and progression of these conditions. Some studies demonstrated that, with chlamydia infection, susceptibility and inflammatory responses in the female genital tract were regulated by sex hormones [2]. MacDonald et al. also found that hormones could modify the susceptibility of human primary genital epithelial cells to herpes simplex virus in females [3]. Moreover, women, especially pregnant women, at the luteal phase of the menstrual cycle when progesterone levels are highest, are most susceptible to Candida albicans infections [4]. It was also found that, in women, the susceptibility to ascending gonococcal infection changes with menstrual cycle. Our previous studies showed that progesterone delayed the onset of apoptosis in polymorphonuclear leucocytes (PMNs) 
challenged by Neisseria gonorrhoeae (Ngo) in vitro [5]. Furthermore, progesterone could inhibit the mRNA expression of inflammatory factors such as iNOS, TNF- $\alpha$, and IL$1 \beta$. On the basis of these findings, we postulate that progesterone may be related to the absence of symptoms of gonorrheal infections in females. Progesterone, progesteronerelated nitric oxide (NO), and the progesterone productionrelated enzymes may be implicated in the mechanism.

Besides hormones, the status of the wild-type strains per se is presumably an important factor leading to the presence or absence of the symptoms. The virulence of Ngo varies with their genotypes, and it directly determines the intensity of inflammatory responses of organisms to Ngo. On the other hand, progesterone influences not only the inflammatory response of organisms to Ngo, but also the virulence and other biological properties of Ngo [6,7]. For example, progesterone may affect the mtrCDE efflux system of some Ngo, thereby, changing their survival ability [8]. This study was designed to examine the relation between the presence of symptoms and the serum progesterone level in patients with gonorrheal infection.

\section{Materials and methods}

\section{Isolation of $N$. gonorrhoeae}

N. gonorrhoeae were collected from 975 female patients aged 18 to 42 years who visited the sexually transmitted disease (STD) clinics of Union Hospital and Tongji Hospital of Tongji Medical College, Huazhong University of Science and Technology, Wuhan, China, from February 2006 to March 2008. Isolates were inoculated onto nonselective or selective media, and, when necessary, subjected to microcultures. Ngo were identified by employing the oxidase test and Gram-staining, and finally confirmed by using coagglutination (Phadebact or specific monoclonal antibodies) $[9,10]$. The symptomatic group was defined if both of the following were observed: (1) easily induced endocervical bleeding or mucopurulent endocervical exudate were present on examination and (2) 30 or more PMN/ HPF were present on Gram stain smear of endocervical secretions. The asymptomatic group did not develop obvious symptoms, but cervical hypertrophy and erosion were found in gynecological examination and 10 30 PMN/HPF were present on Gram stain smear of endocervical secretions. In the normal group, less than 10 PMN/HPF were found on Gram stain smear of endocervical secretions [11, 12].

\section{Hormone assay}

Blood was drawn from all of the subjects on an empty stomach. The blood was centrifuged at $3,000 \mathrm{~g}$ for $15 \mathrm{~min}$ at $4^{\circ} \mathrm{C}$, and the supernatant was harvested and stored at $-70^{\circ} \mathrm{C}$. Serum estradiol and progesterone were measured by radioimmunoassay (RIA). RIA kits were provided by Shenzhen Larewen Biomedical Engineering Technology Co. Ltd., China. The assay sensitivity was $10 \mathrm{pg} / \mathrm{ml}$ for estradiol and was between $0.2 \sim 0.6 \mathrm{ng} / \mathrm{ml}$ for progesterone. The inter- and intra-assay coefficients of variation were all $<10 \%$.

Strain culture and characterization

The bacteria were cultured on Thayer-Martin agar and GCbased chocolate agar, and identified by Gram staining and oxidase test. Pure cultures supplemented with $8 \%$ skimmed milk and $10 \%$ fetal calf serum were used for long-term storage at $-70^{\circ} \mathrm{C}$. The strains had not been passaged prior to this study. Auxotyping and serotyping were not done on the strains. For DNA analysis, strains were retrieved from the stock and cultured on GC-based chocolate agar overnight at $36^{\circ} \mathrm{C}$ in $5 \% \mathrm{CO}_{2}$. A single colony was subcultured once before the DNA was prepared. In brief, bacterial suspensions (OD at $540 \mathrm{~nm}$ of $1.0 \sim 2.0 ; \sim 10^{8}$ $10^{9} \mathrm{cfu} / \mathrm{mL}$ ) were made in $0.17 \mathrm{~mol} / \mathrm{L} \mathrm{PBS}(\mathrm{pH} 7.3)$, and the bacteria were pelleted by centrifugation at $2,000 \mathrm{~g}$ for $5 \mathrm{~min}$, washed once, resuspended in PBS, and boiled for $5 \mathrm{~min}$. The lysate was centrifuged at 2,000g for $5 \mathrm{~min}$, and the supernatant was stored at $-20^{\circ} \mathrm{C}$. All positive isolates were genotyped by using NG-MAST as described previously $[13,14]$. DNA sequencing of $p o r-t b p B$ was carried out by Shanghai Sangon Biological Engineering Technology \& Services Co. Ltd., China. Alignments of trimmed sequences were performed by the use of ClustalX (version 1.8). Gonococcal genetic island (GGI) positive-specific and GGI negative-specific polymerase chain reaction (PCR) amplification was performed on all strains examined. Chromosomal DNA of each strain was amplified using primers $77 \mathrm{~F}$ (5'-TAACAGCAGACGCTCCATTC-3') and 86R (5'-CAAGCGCATGGTACATGAAT-3') (Tm $57^{\circ} \mathrm{C}$; extension time $90 \mathrm{~s})$ and primers $73 \mathrm{~F}$ (5'-AGCCATCAGG GAGGCGGATA-3') and hlh-ggiR (5'-CAGGCAAA CAGCTATTTGAG-3') (Tm $54^{\circ} \mathrm{C}$; extension time $90 \mathrm{~s}$ ).

\section{Statistical analysis}

The Statistical Package for Social Sciences (SPSS v13.0 for windows) was used for the data analysis. Descriptive statistics were performed for each variable. Quantitative results are presented as mean $( \pm \mathrm{SD})$. the means were compared by using one-way analysis of variance (ANOVA) and the two-sample $t$-test. Proportions for the two groups were compared by using the $\chi^{2}$ test and the Fisher exact test. A $p$-value $<0.05$ was considered to be statistically significant. 
Table 1 Serum progesterone and estradiol levels in the two different groups $(\overline{\mathrm{x}} \pm \mathrm{s})(\mathrm{pg} / \mathrm{ml})$

\begin{tabular}{lll}
\hline Groups & Symptomatic & Asymptomatic \\
\hline$n$ & 42 & 39 \\
Progesterone & $0.65 \pm 0.32 \mathrm{ng} / \mathrm{mL}^{*}$ & $9.04 \pm 4.95 \mathrm{ng} / \mathrm{mL}$ \\
Estradiol & $87.24 \pm 7.06 \mathrm{pg} / \mathrm{mL}$ & $119.35 \pm 6.45 \mathrm{pg} / \mathrm{mL}$ \\
\hline
\end{tabular}

Comparison of the symptomatic group and the asymptomatic group, $* p<0.01$

\section{Results}

Serum estradiol and progesterone levels

The serum progesterone and estradiol levels were measured in the three groups. Comparison of the symptomatic group and the asymptomatic group showed that serum progesterone levels of the asymptomatic group were significantly higher $(p<0.01)$ than those of the symptomatic group. However, no significant difference was found in the serum estradiol levels between the symptomatic group and its asymptomatic counterpart (Table 1). We found that the asymptomatic subjects were mostly at the luteal phase, while the subjects in the symptomatic group were mainly at the follicular or ovulatory phase.

\section{Phenotypic and genotypic characterization of isolates}

Among the 975 women enrolled in this study, 91 women $(9.33 \%)$ were diagnosed with gonococcal infection and in ten women, specimens were not collected. A total of 81 isolates were obtained. In 39 women, the symptoms were not apparent, but cervical hypertrophy and erosion were found in gynecological examination (asymptomatic group). Eighty-one isolates showed 41 and 30 divergent partial NGMAST por and $t b p B$ alleles, respectively, which resulted in the assignment of 50 different sequence types (STs). The genotypic characterization of ST isolates (total 81 strains) is summarized in Table 2. ST2951 was the most prevalent ST found in asymptomatic patients. Among them, 7 of 8 strains of the ST2951 genotype were from the asymptomatic group.

\section{Discussion}

Strong evidence demonstrated that sex steroids influence the pattern of inflammatory substances in human. The role of progesterone as a natural immune-suppressant has been known for a long time. The higher incidence of immunemediated diseases in women has been attributed to progesterone down-regulating proinflammatory cytokines in cross-sectional trials [15-17]. Progesterone may be of particular relevance in female asymptomatic gonococcal infections and need to be further investigated. Hedges et al. conducted an in vivo experiment and found that women with gonococcal cervicitis did not exhibit elevated local levels of IL-1, IL-6, and IL-8 [18]. Their study, however, did not to take the influence of hormones into account. In this study, we demonstrated that, in females with gonococcal infection, the levels of serum progesterone were significantly higher in asymptomatic patients than in symptomatic patients $(p<0.01)$. In combination with our previous studies, our present results suggest that progesterone may play a role in female asymptomatic infections by down-regulating inflammatory response and delaying the onset of apoptosis. The levels of serum progesterone were higher in the asymptomatic group than in the symptomatic group. The reason for this may be that the serum was collected from asymptomatic subjects mostly at the luteal phase, while in the symptomatic group, the serum was taken mainly at the follicular phase.

Variation within the por and $t b p B$ loci is likely to be selected by the human immune response, and there are large numbers of alleles at both loci [19]. The internal fragments of por and $t b p B$ from 81 isolates were amplified, and both strands were sequenced. There were 41 different por alleles and 30 different $t b p B$ sequences, resulting in 50 different two-locus allelic profiles. Among the 81 isolates, the most common alleles were por-217 and $t b p B-33$, which were found in 10 and 13 isolates, respectively. Only in one isolate were 30 por alleles and $19 t b p B$ alleles found. ST2951, ST735, and ST436 were common in asymptomatic patients, but ST809 and ST369 was prevalent in symptomatic patients. This subpopulation of Ngo prevails in females with asymptomatic infections in our series

Table 2 The no-unique Neisseria gonorrhoeae multi-antigen sequence types (NG-MAST) associated with female infections in Wuhan, China

\begin{tabular}{|c|c|c|c|c|c|c|}
\hline \multirow[t]{2}{*}{ Groups } & \multicolumn{3}{|c|}{ Collected time } & \multirow[t]{2}{*}{ No-unique ST types (por allele-tbpB allele) } & \multicolumn{2}{|l|}{ GGI } \\
\hline & Luteal phase & Follicular phase & Uncertain & & Positive & Negative \\
\hline Asymptomatic & 27 & 9 & 3 & $\begin{array}{l}2951(217-679), 735(242-4), 436(90-21), 2943 \\
(1226-33), 314(241-75), 147(53-21)\end{array}$ & 27 & 12 \\
\hline Symptomatic & 16 & 25 & 1 & $\begin{array}{l}809(543-33), 369(256-137), 2948(4-8), 421 \\
(206-156), 641(90-186)\end{array}$ & 29 & 13 \\
\hline
\end{tabular}

ST, sequence type 
(Wuhan) now. In Sweden, the genetic characterization identified one widely spread ciprofloxacin-resistant $N$. gonorrhoeae ST147 strain [20]. The ST147 strain successively appeared in America and Africa. It is the most reported genotype of $N$. gonorrhoeae in the literature at present. We obtained only three isolates of the ST147 strain. After comparison, we know that NG-MAST sequence types might be related to the asymptomatic gonococcal infections, but the connection remains unknown.

The GGI, present in $80 \%$ of gonococcal strains, contains two lytic transglycosylase homologs, AtlA and $\mathrm{LtgX}$, which may produce peptidoglycan (PG) fragments [21]. PG fragments can induce IL- 8 production and cause the infiltration of PMNs to the sites of infection. $N$. gonorrhoeae can survive in PMNs and infect other individuals through purulent secretion. It was presumed that Ngo mutants defective in the type IV secretion system may induce less IL-8 production and less PMN infiltration. Table 2 shows that both GGI-positive and GGI-negative strains were found in the asymptomatic group and there were GGI-positive and GGI-negative strains in the symptomatic group, suggesting that GGI has nothing to do with female asymptomatic gonococcal infection.

In conclusion, our results showed that the Ngo GGI genotype is not linked with female gonococcal infections, but progesterone is associated with the outcome of the condition. A better understanding of the mechanisms of female asymptomatic gonococcal infections can help to improve therapeutic and diagnostic results of the disease. The molecular mechanism of the interaction between progesterone and PMNs in gonorrhea warrants further investigation.

Acknowledgments This project was supported by grants from the National Natural Sciences Foundation of China (no. 30700717) and the Research Fund for the Doctoral Program of Higher Education of China (no. 20070487140).

Open Access This article is distributed under the terms of the Creative Commons Attribution Noncommercial License which permits any noncommercial use, distribution, and reproduction in any medium, provided the original author(s) and source are credited.

\section{References}

1. Edwards JL, Apicella MA (2004) The molecular mechanisms used by Neisseria gonorrhoeae to initiate infection differ between men and women. Clin Microbiol Rev 17:965-981

2. Kaushic C, Zhou F, Murdin AD et al (2000) Effects of estradiol and progesterone on susceptibility and early immune responses to Chlamydia trachomatis infection in the female reproductive tract. Infect Immun 68:4207-4216
3. MacDonald EM, Savoy A, Gillgrass A et al (2007) Susceptibility of human female primary genital epithelial cells to herpes simplex virus, type-2 and the effect of TLR3 ligand and sex hormones on infection. Biol Reprod 77:1049-1059

4. Fidel PL Jr, Cutright J, Steele C (2000) Effects of reproductive hormones on experimental vaginal candidiasis. Infect Immun 68:651-657

5. Chen H, Wu Z, Li J, Chen R et al (2009) Effect of progesterone on gonococci-induced apoptosis and respiratory burst of human polymorphonuclear leukocytes in vitro. Int $\mathrm{J}$ Dermatol 48 (9):1011-1016

6. Morse SA, Fitzgerald TJ (1974) Effect of progesterone on Neisseria gonorrhoeae. Infect Immun 10:1370-1377

7. Lysko PG, Morse SA (1980) Effects of steroid hormones on Neisseria gonorrhoeae. Antimicrob Agents Chemother 18:281288

8. Jerse AE, Sharma ND, Simms AN et al (2003) A gonococcal efflux pump system enhances bacterial survival in a female mouse model of genital tract infection. Infect Immun 71:5576-5582

9. Paine TC, Fenton KA, Herring A et al (2001) GRASP: a new national sentinel surveillance initiative for monitoring gonococcal antimicrobial resistance in England and Wales. Sex Transm Infect 77:398-401

10. Fenton KA, Hughes G, Herring A et al (2004) The Gonococcal Resistance to Antimicrobials Surveillance Programme: Protocol 2004 Collection. Health Protection Agency Centre for Infections, London

11. Marrazzo JM, Handsfield HH, Whittington WL (2002) Predicting chlamydial and gonococcal cervical infection: implications for management of cervicitis. Obstet Gynecol 100:579-584

12. Bozicevic I, Fenton KA, Martin IM et al (2006) Epidemiological correlates of asymptomatic gonorrhea. Sex Transm Dis 33:289295

13. Palmer HM, Young H, Martin IM et al (2005) The epidemiology of ciprofloxacin resistant isolates of Neisseria gonorrhoeae in Scotland 2002: a comparison of phenotypic and genotypic analysis. Sex Transm Infect 81:403-407

14. Moodley P, Martin IM, Pillay K et al (2006) Molecular epidemiology of recently emergent ciprofloxacin-resistant Neisseria gonorrhoeae in South Africa. Sex Transm Dis 33:357-360

15. Cutolo M, Sulli A, Capellino S et al (2004) Sex hormones influence on the immune system: basic and clinical aspects in autoimmunity. Lupus 13:635-638

16. Kaushic C, Ashkar AA, Reid LA et al (2003) Progesterone increases susceptibility and decreases immune responses to genital herpes infection. J Virol 77:4558-4565

17. Brooks-Asplund EM, Tupper CE, Daun JM et al (2002) Hormonal modulation of interleukin-6, tumor necrosis factor and associated receptor secretion in postmenopausal women. Cytokine 19:193-200

18. Hedges SR, Mayo MS, Mestecky J et al (1999) Limited local and systemic antibody responses to Neisseria gonorrhoeae during uncomplicated genital infections. Infect Immun 67:3937-3946

19. Martin IM, Ison CA, Aanensen DM et al (2004) Rapid sequencebased identification of gonococcal transmission clusters in a large metropolitan area. J Infect Dis 189:1497-1505

20. Unemo M, Sjöstrand A, Akhras M et al (2007) Molecular characterization of Neisseria gonorrhoeae identifies transmission and resistance of one ciprofloxacin-resistant strain. APMIS 115:231-241

21. Hamilton HL, Domínguez NM, Schwartz KJ et al (2005) Neisseria gonorrhoeae secretes chromosomal DNA via a novel type IV secretion system. Mol Microbiol 55:1704-1721 